\title{
Typical Daily Power Curve Mining for Energy Storage Systems under Smoothing Power Fluctuation Scenarios
}

\author{
Xiyun Yang, ${ }^{1}$ Jie Ren, ${ }^{2}$ Xiangjun Li $\left(\mathbb{D},{ }^{3}\right.$ and Hang Zhang ${ }^{1}$ \\ ${ }^{1}$ School of Control and Computer Engineering, North China Electric Power University, Beijing 102206, China \\ ${ }^{2}$ School of Electrical Engineering, Shandong University, Jinan 250061, China \\ ${ }^{3}$ The State Key Laboratory of Control and Operation of Renewable Energy and Storage Systems, \\ China Electric Power Research Institute, Beijing 100192, China
}

Correspondence should be addressed to Xiangjun Li; lirr0901@126.com

Received 8 August 2017; Revised 17 December 2017; Accepted 1 January 2018; Published 30 January 2018

Academic Editor: Emilio Jiménez Macías

Copyright (C) 2018 Xiyun Yang et al. This is an open access article distributed under the Creative Commons Attribution License, which permits unrestricted use, distribution, and reproduction in any medium, provided the original work is properly cited.

Under the application scenario of smoothing photovoltaic (PV) power fluctuation, a novel typical daily power curve mining method is developed for a battery energy storage system (BESS) that utilizes the power probability distribution and Bloch spherical quantum genetic algorithm. The charging/discharging of BESS is analyzed by applying fuzzy-c means clustering techniques. In the mining approach, at any sample time, those distribution intervals containing concentrated power points are individually located by using probability distribution information and Bloch spherical quantum genetic algorithm. Character power for the specified interval can also be determined using Bloch spherical quantum genetic algorithm. Next, a roulette principal is employed, to determine one value from the character power data as a typical value of the mined power curve at the sample time. By connecting the typical power at each sample time, the typical daily power curve for BESS is achieved. Based on typical power curve, decision-maker can master the important operating parameters of BESS and analyze optimal capacity allocation. By error evaluation indexes between the mined typical daily power curve and power curve under different weather patterns, the simulation results verify that the mined power curve can address the operating power of the BESS under different weather patterns.

\section{Introduction}

Photovoltaic (PV) power generation technology has grown tremendously in recent years [1]. PV conversion technology is discontinuous and fluctuating, due to the variety of solar radiation. It has caused severe challenges for the reliable and safe operation of power grids, especially at high levels of penetration $[2,3]$. It is well known that the power fluctuation can be mitigated through deployment of energy storage systems, alongside PV power generation.

Electric energy can be stored electromagnetically, kinetically, or as potential energy, such as supercapacitors, flywheels, batteries, compressed air energy storage, and hydropumped storage based on different storage methods [4]. Among various energy storage options, the battery energy storage system (BESS) is widely applied for easy implementation via charging/discharging of electric energy. BESS is an attractive technology to solve problems concerning the high penetration of PV stations. Based on prior studies, a number of approaches to optimizing the capacity of BESS are reported from several aspects, such as better utilization of PV [5] and reduction of generation costs [6]. The technical and economic model of the PV-battery [7] and lifecycle-based cost modeling for batteries [8] are also investigated. In the field of operation control, a power management mechanism for grid PV with BESS is designed [9]. An optimization scheme for an energy dispatch schedule is presented in [1012]. These studies mainly focused on the suitable capacity allocation, the minimum economic cost, and optimizing operation control for PV-battery system. The research shows that there is little research on mining the typical daily power curve of BESS. Since a large-scale BESS is highly expensive, it is valuable that the typical operation power curve of BESS be mined from the massive operation power data in BESS. As we know, electric load curve can provide useful information. Using the typical daily power curve extracted under 
different operational conditions, valuable knowledge can be obtained from analyzing the charging/discharging of BESS, including maximum charging/discharging power, maximum power fluctuation rate, and the charging/discharging capacity in one day. Typical daily power curve can not only help understand the operational features of the PV-battery system to optimize performance of BESS, but also provide useful data to easily calculate the optimal capacity allocation of BESS for photovoltaic power station to save economic cost.

The mining technology of the typical load curve is often concerned with electric load. Methods to address this problem consist of statistical methods and artificial intelligence based techniques. Artificial intelligence methods include fuzzy logic approaches [13], neural networks [14], expert systems [15], and pattern-recognition techniques [1618]. Statistical methods include linear regression, exponential smoothing [19], stochastic time series [20], and state space models [21].

At present, there is little research on load curve for BESS with renewable generation systems. In this work, we study the mining typical daily power curve of BESS under the application scenario of smoothing PV power fluctuations. After analyzing the characteristics of BESS power, we present a novel mining method of typical daily power curve for BESS, considering the probability distribution of power among scattered historical power time series. For any sample time, a Bloch spherical quantum genetic algorithm is adopted, to locate distribution interval ranges containing denser power points from the vertical time series of power data (the power data at the same time across different days). Character power mapping of every distribution interval is obtained by the Bloch spherical quantum genetic algorithm. Next, a roulette principal is employed, to determine one power value from a group of character power data as the typical power data for this sample time. Finally, connecting the typical power values at every sample time, a typical daily power curve for BESS is generated and error evaluation indexes are also compared.

The remainder of this paper is organized as follows. The characteristics of operation for BESS under different weather patterns are presented in Section 2. In Section 3, the mining algorithm of the typical daily power curve is developed based on probability distribution and Bloch spherical quantum genetic algorithm. In Section 4, the proposed strategy is applied to a case study system with real PV station data. Finally, conclusions are given in Section 5.

\section{Operation Characteristics of Charging/Discharging Power in BESS}

As solar irradiation intensity varies with different weather, the PV power also presents different fluctuation and further causes a variety of charging/discharging power in BESS. In order to discover the operating characteristics of BESS, a clustering method is employed to classify the historical data.

2.1. The Fuzzy-C Means Clustering Techniques for PV Power Data. Clustering is a useful method to classify a set of data into groups with similar attributes. Fuzzy-C means (FCM) is one of the most popular clustering techniques. In this work, a FCM is employed to cluster the PV power data. We use the objective function of the FCM method [22] to minimize the distance between data points and center of clusters:

$$
J(U, V)=\sum_{i=1}^{M} \sum_{c=1}^{C}\left(\mu_{c}(i)\right)^{m}\left\|X_{i}-V_{c}\right\|^{2} .
$$

$J(U, V)$ is the object function, $\mathbf{X}=\left\{X_{1}, X_{2}, \ldots, X_{M}\right\}$ is input data to be clustered, $M$ is the total number of input data, $C$ is the total number of clusters, $\mathbf{V}=\left\{V_{1}, V_{2}, \ldots, V_{c}\right\}$ is the matrix of cluster centers, $\mu_{c}(i)$ is membership degree of the $i$ th input data to center of the Cth cluster, and $m$ is the userdefined parameter termed "amount the index" of fuzziness. The procedure of FCM for PV power is briefed as follows: classify the weather pattern into six types: sunny, cloudy, overcast, rain/snow, cloudy to sunny, and sunny to cloudy; that is, $C=6$. Selecting the irradiance fluctuation rate, maximum irradiance ratio, and temperature as clustering characters, the input data is expressed as

$$
X_{i}=\left[V_{1 i}, V_{2 i}, K_{i}, T_{i}\right] \text {. }
$$

For the $i$ th day, $V_{1 i}$ is the irradiance fluctuation rate before 12 hours, $V_{2 i}$ is the irradiance fluctuation rate after 12 hours, $T_{i}$ is average temperature, and $K_{i}$ is the maximum irradiance ratio.

The maximum irradiance ratio $K_{i}$ is the ratio between the actual solar irradiance received of PV panel $I_{i}$ and maximum irradiance in external horizontal plane $E_{i}^{c}$ at the same sample time. It is calculated as

$$
K_{i}=\frac{I_{i}}{E_{i}^{c}} .
$$

The irradiance fluctuation rate $V_{i}$ is the irradiance fluctuation on the PV panel in $i$ th day. The formula is

$$
V_{i}=\frac{\sum_{k=2}^{n} \sqrt{\left(I_{k, i}-I_{k-1, i}\right)^{2}+\Delta t^{2}}}{\sum_{k=2}^{n} \sqrt{\left[\left(E_{k, i}^{c}-E_{k-1, i}^{c}\right) K_{i}\right]^{2}+\Delta t^{2}}} .
$$

$I_{k, i}$ is the actual surface irradiance in the horizontal plane, $E_{k, i}^{c}$ is maximum irradiance in external horizontal plane, and $\Delta t$ is the sample time.

FCM is an iterative algorithm. First, the initial membership value must meet requirement (5). Secondly, according to (6), the vector of cluster center for iteration $k$ can be computed:

$$
\begin{aligned}
\sum_{c=1}^{C} \mu_{c}(i) & =1, \quad \forall i \in 1, \ldots, M \\
V_{c} & =\frac{\sum_{i=1}^{M}\left(\mu_{c}(i)^{(k)}\right)^{m} X_{i}}{\sum_{i=1}^{M}\left(\mu_{c}(i)^{(k)}\right)^{m}}, \quad c=1, \ldots, C .
\end{aligned}
$$

The membership value is updated by (7) for each iteration.

$$
\mu_{c}(i)^{(k)}=\frac{1}{\sum_{j=1}^{C}\left[\left\|X_{i}-V_{c}\right\| /\left\|X_{i}-V_{j}\right\|\right]^{2 /(m-1)}} .
$$


The iteration continues until terminal condition (8), where $\varepsilon$ is an approximately small value.

$$
\left\|\mu_{c}(i)^{(k)}-\mu_{c}(i)^{(k-1)}\right\|<\varepsilon .
$$

2.2. Operation Characteristic of BESS. The PV actual output power of the solar station was applied with $30 \mathrm{MW}$ capacity in China using the FCM method to classify the data. The sampling time was one minute. According to the requirement of the fluctuation rate of active power for the PV station, the charging/discharging power in BESS under the different weather patterns was obtained by utilizing a smoothing strategy based on slope control [23]. The curves of PV power, the power of BESS, and the power after smoothing under six weather patterns are shown in Figure 1.

It can be seen from Figure 1 that BESS power has different features among various weather patterns. On sunny days, the fluctuation of PV power is smaller, and the BESS almost does not discharge and charge power. With the change of the weather, clouds and radiation are fluctuation and the requirement for stored power in BESS to smooth PV power becomes larger. The BESS power is mainly in the range of [-0.2 MW 0.2 MW] on sunny days. During rain or snow, the BESS power is concentrated in the range of $[-2.5 \mathrm{MW}$ $2.5 \mathrm{MW}$. During other weather patterns, the requirements for stored energy in BESS become heavier and the BESS power is mainly various in the range of [-8 MW $8 \mathrm{MW}]$.

In order to discover operation aspects detailed in BESS under the different weather patterns, probability statistical information including the distribution histogram and fitting curve of probability density for BESS power is shown in Figure 2. Since the output power in the PV station is zero during the night or times with lower solar irradiance, only power data within 6:00-19:00 is counted. It can be seen from Figure 2 that the fitting curves of probability density stretch away from zero under worse weather patterns. This trend illustrates that the power requirements for stored energy become heavier due to the larger fluctuations of PV power in worse weather.

\section{Mining Method of Typical Daily Power Curve for BESS}

3.1. The Mining Algorithm for Typical Power Curve. Since the fluctuation of PV power varies for different weather, there are scattered data of BESS power at the same sample times across different days. In order to mine typical daily power curves of BESS under smoothing PV power fluctuation, we develop the mining algorithm as follows:

(1) For $k$ time instant, a typical power value $P_{k}$ mined among historical power data should approximately describe the charging/discharging feature of BESS during different days.

(2) Integrating typical power values at every sampling time instant, a typical daily power curve of BESS can be accomplished as follows: $P_{\text {typical }}=\left[P_{1}, P_{2}, \ldots, P_{n}\right]$, where $n$ is the number of sample points for one day.
A key issue is to determine the typical power value $P_{k}$ at time $k$. Since the power data of BESS is very dispersed at time $k$ across different days, it is difficult to automatically extract suitable typical power for time $k$ from large amounts of data. In this work, a novel idea considering data frequency information is proposed to mine data. A mining algorithm is put forward to extract the typical power value for time $k$ by utilizing the probability distribution information of power data in the vertical time series and Bloch spherical quantum genetic algorithm. The vertical time series are constructed with historical power data at the same time $k$ during different days. First, a Bloch spherical quantum genetic algorithm is adopted to search the distribution interval range containing the densest power points for time $k$ from the vertical time series. Because there exist many power points in searched distribution range, a Bloch spherical quantum genetic algorithm is continually employed to locate one power value from many power points as character power mapping to this interval. Although this extracted character power value occurs at higher frequencies for time $k$, it is still inaccurate or incomplete to only utilize this character power as suitable typical power for time $k$. Thus in our work, for the remaining data, we iterate this process until every distribution interval containing more power points and character power is found using the terminal condition: there is no distribution intervals range where the statistical probability of power points belonging to the interval is higher than the set threshold $\varepsilon$. At this time, we have found $N$ distribution intervals, meaning that there exist $N$ character power values for sample time $k$. We use these $N$ character power data to construct a character power vector, and record the frequency probability of distribution-interval-mapped character power at time $k$. We then employ the roulette principle based on the probability to determine one character power as typical power from the character power vector for sample time $k$. The roulette principle has an advantage of maintaining diversity of the typical power.

3.2. The Step of Mining Algorithm of Typical Power Curve. Applying the above principle and using the mining typical power value at time $k$ as an example, the detailed step for extracting the load curve for a BESS from its operational data is as follows.

Step 1. Read BESS power data during $m$ days, and construct power matrix $\mathbf{M}$, with the element of $\mathbf{M}$ representing the BESS power historical data during $m$ days. There are $n$ sample points for one day.

$$
\mathbf{M}_{m \times n}=\left[\begin{array}{c}
p_{11}, p_{12}, \ldots, p_{1 n} \\
p_{21}, p_{22}, \ldots, p_{2 n} \\
\vdots \\
p_{m 1}, p_{m 2}, \ldots, p_{m n}
\end{array}\right] .
$$

Step 2. Extract power vector $\mathbf{P}_{k_{1}}$ at $k$ time during $m$ days: $\mathbf{P}_{k 1}=\left[p_{1 k}, p_{2 k}, \ldots, p_{m k}\right]^{T}$. 

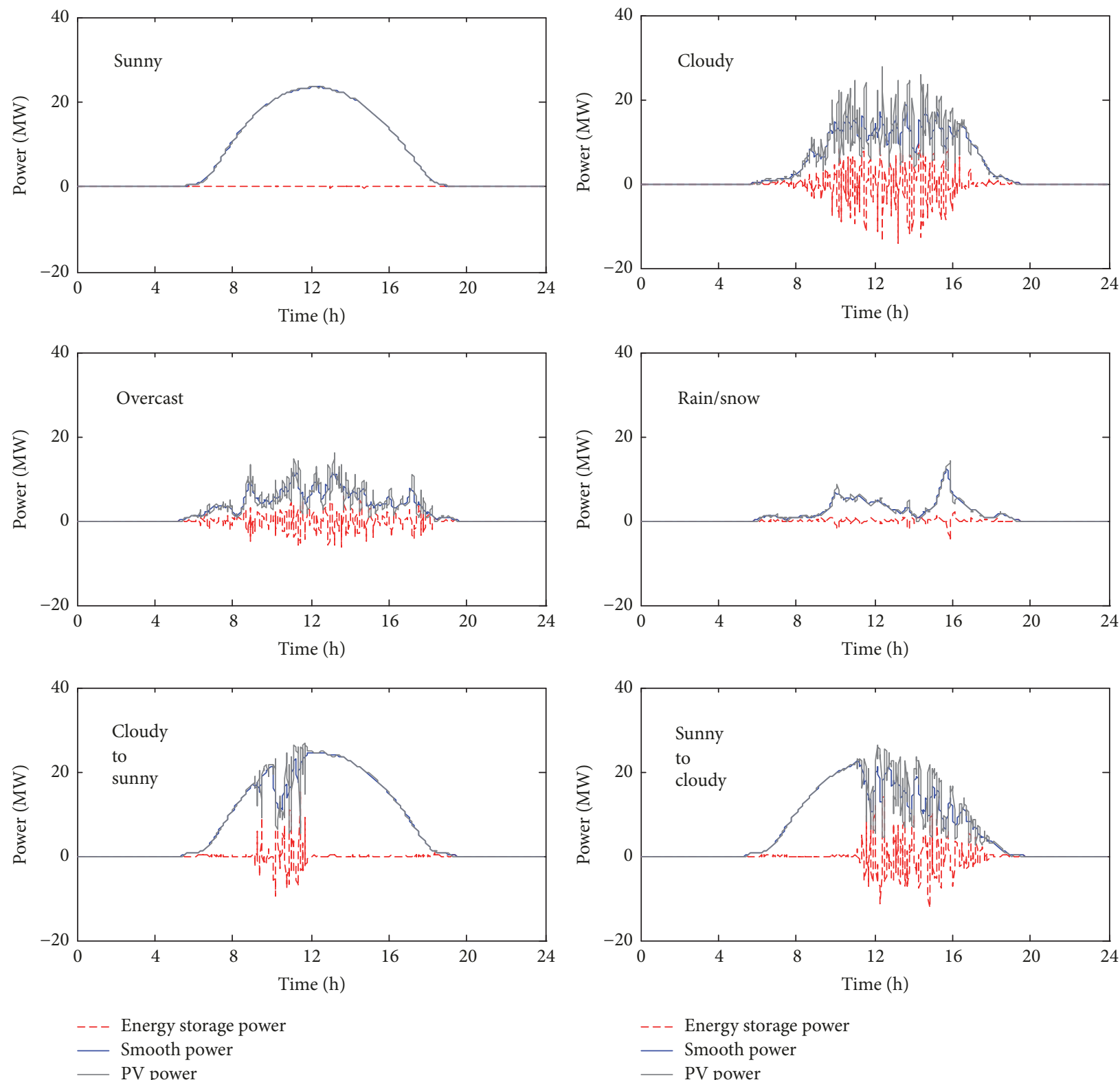

FIgURE 1: Power curves under typical weather patterns.

For example, assuming that $k$ time is equal to 9:00 in the morning, we sample the power data at 9:00 am across ten days to construct vertical time series data.

Step 3. Search the distribution interval containing the most concentrated power points $L_{\mathrm{opt} 1}=\left[\begin{array}{ll}x_{\mathrm{opt} 1} & x_{\mathrm{opt} 1}+d\end{array}\right]$ using the Bloch spherical quantum genetic algorithm.

The process can be briefly discussed as follows.

If the lower limit of the interval is $x$ and the range of interval is $d$, then the distribution interval is $L=\left[\begin{array}{ll}x & x+d\end{array}\right]$, where $d$ is a constant according to experience.

$$
\max J=\max \sum_{g=1}^{m} f\left(p_{g k}\right)
$$

$$
f\left(p_{g k}\right)= \begin{cases}1, & x \leq p_{g k} \leq x+d \\ 0, & \text { else }\end{cases}
$$

where $p_{g k}$ denotes an element of $\mathbf{P}_{k_{1}}, g=1,2, \ldots, m$.

Thus, the first distribution interval containing the most concentrated power points is obtained as $L_{\text {opt1 }}=$

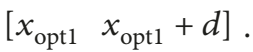

Step 4. Calculate the frequency probability $F_{k 1}$ of the distribution interval.

Extract all power values in the distribution interval $L_{\text {opt1 }}$, to construct the interval power vector $\mathbf{I}_{k 1}=$ $\left[p_{1 k}^{\prime}, p_{2 k}^{\prime}, \ldots, p_{i k}^{\prime}\right]^{T}, 1<i \leq m$, where $p_{i k}^{\prime}$ denotes the power 

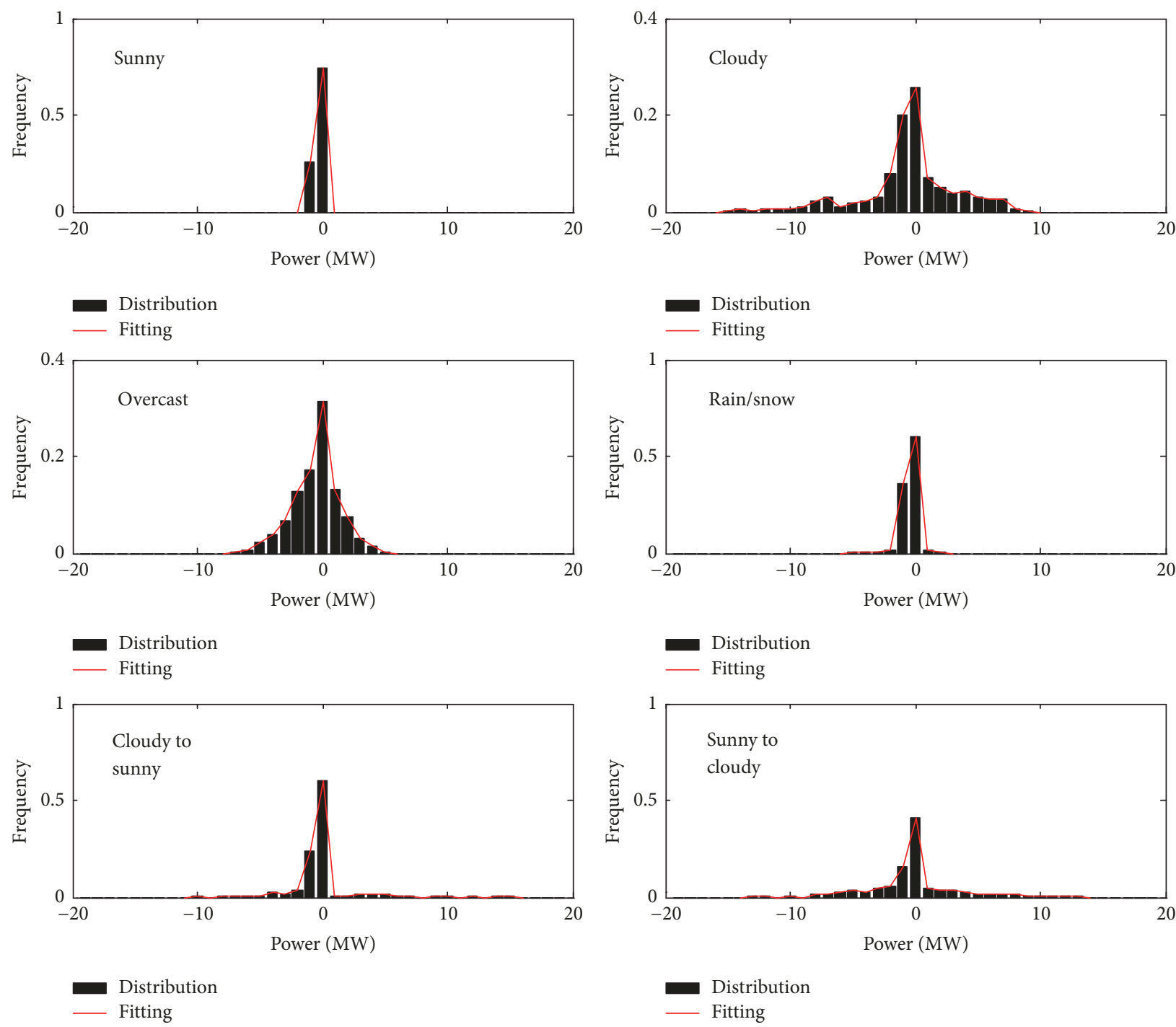

FIgURE 2: Power distribution and fitting curve for BESS.

value in $L_{\mathrm{optl}}$. This can be diagramed as encircling data set in Figure 3(a).

We calculate the frequency probability $F_{k 1}$ of distribution interval as

$$
F_{k 1}=\frac{t}{m}
$$

where $t$ is the size of the interval power vector $\mathbf{I}_{k 1}$.

Step 5. Search the character power $E_{k 1}$ mapping in the previously specified interval by Bloch spherical quantum genetic algorithm.

We define $p_{\min }$ as the minima among the elements of $\mathbf{I}_{k 1}$ and $p_{\max }^{\prime}$ as the maxima among the elements of $\mathbf{I}_{k 1}$. Since character power $E_{k 1}$ represents typical power value within the distribution, the power value, which has the nearest Euclid distance to other elements of $\mathbf{I}_{k 1}$ in the range $\left[p_{\min }^{\prime} p_{\max }^{\prime}\right]$, can be considered as character power. The Bloch spherical quantum genetic algorithm is used in the range $\left[\begin{array}{ll}p_{\min }^{\prime} & p_{\max }^{\prime}\end{array}\right]$ to obtain the character power. Optimizing the object function $T$ is defined as

$$
\min T=\min \sqrt{\sum_{j=1}^{t}\left(E_{k 1}-p_{j k}^{\prime}\right)^{2}} .
$$

Step 6. Remove the interval power vector $\mathbf{I}_{k 1}$ from power vector $\mathbf{P}_{k_{1}}$ and create new power data $\mathbf{P}_{k_{2}}$. Iterate Steps 3 and 4 to get the next optimal distribution interval $L_{\text {opt2 }}$ and the interval power vector $\mathbf{I}_{k 2}$. This can be diagramed as the encircling set in Figure 3(b). Calculate the frequency probability $F_{k 2}$.

Step 7. If $F_{k 2}$ is smaller than the threshold $\varepsilon$, then go to Step 8. Else, iterate Step 6 to calculate the character power $E_{k 2}$ and find the overall character power, until the frequency probability $F_{k i}$ is smaller than the threshold. Following this, go to Step 8. Threshold $\varepsilon$ can be determined according to the 


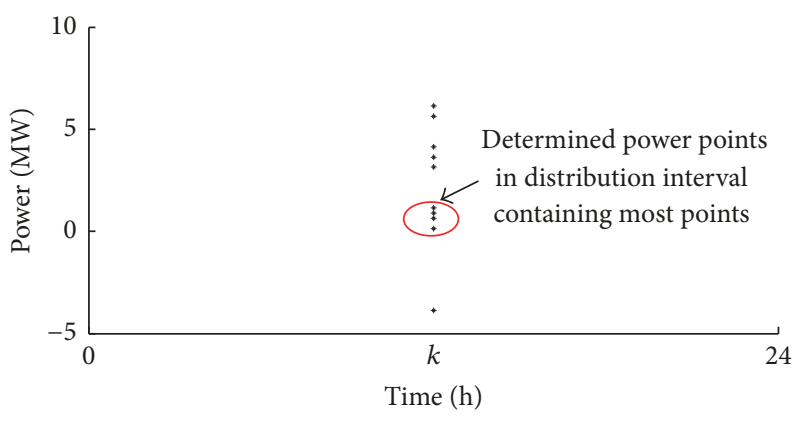

(a)

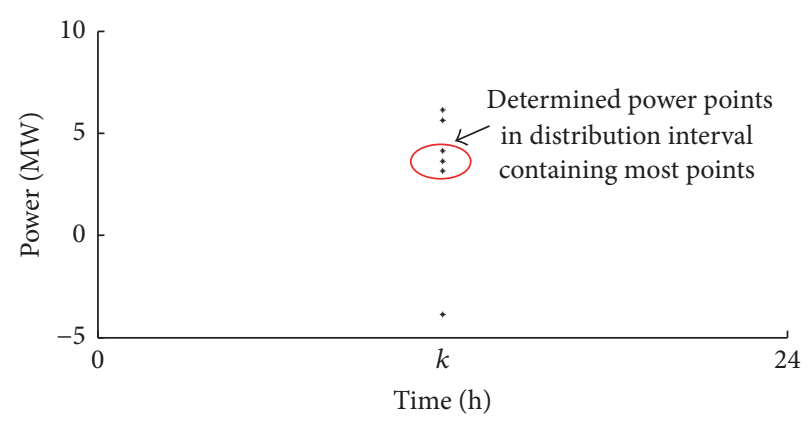

(b)

FIGURE 3: Schematic diagram of mining specified distribution intervals: (a) interval power vector mapping distribution interval containing the most power points in the first search; (b) interval power vector mapping distribution interval containing the most power points in the second search.

dispersive degree of the power point. If the power point is scattered, a small value is assigned to the threshold $\varepsilon$. When the power value is concentrated, the threshold $\varepsilon$ can be of a large value.

Step 8. Take all character power mapping with a frequency probability larger than threshold $\varepsilon$ to construct the typical character power vector $\mathbf{E}_{k}=\left[E_{k 1}, E_{k 2}, \ldots, E_{k h}\right]$ at time $k$. Applying the principle of roulette based on the probability of character power, only one element, $E_{k i}$, is selected as the typical power $P_{k}$ in BESS at time $k$. Roulette has an advantage of maintaining the most diverse selected typical power. The probability of character power is calculated as

$$
f\left(P_{k}=E_{k i}\right)=\frac{F_{k i}}{\sum_{i=1}^{h} F_{k i}} .
$$

Therefore, the typical daily power curve is $P_{\text {typical }}=$ $\left[P_{1}, P_{2}, \ldots, P_{n}\right]$, after connecting the overall typical power at all sample times in one day.

Taken as an example, assume that there are ten data sets among historical data at $k$ time: six data sets whose values of discharging power are $0.3 \mathrm{MW}$, one data set whose value of discharging power is $0.31 \mathrm{MW}$, two data sets whose values of discharging power are $0.6 \mathrm{MW}$, and one data set whose value of discharging power is $0.1 \mathrm{MW}$. For the data, a Bloch spherical quantum genetic algorithm first searches the vertical time series data and automatically finds the interval distribution containing the most power points [0.3 MW $0.32 \mathrm{MW}$. Next, a Bloch spherical quantum genetic algorithm can also locate $0.3 \mathrm{MW}$, not $0.31 \mathrm{MW}$, as the character power mapping of the distribution interval. This process is then iterated, with distribution ranges [0.6 MW $0.62 \mathrm{MW}$ ] and [0.1 MW $0.12 \mathrm{MW}$ ] found, and character powers $0.6 \mathrm{MW}$ and $0.1 \mathrm{MW}$ are determined. We choose character powers $0.3 \mathrm{MW}, 0.6 \mathrm{MW}$, and $0.1 \mathrm{MW}$ to generate character power vector [0.3 0.6 0.1]. Finally, the roulette principle is employed to determine the typical power value $P_{k}$ of BESS at time $k$. Character power value $0.3 \mathrm{MW}$ has high probability to become the typical power $P_{k}$ at $k$ time. The roulette principle gives an effective approach to maintaining the diversity of the typical power.
The flow chart of the proposed mining algorithm of the typical daily power curve is illustrated in Figure 4.

\subsection{Optimizing Process Based on the Bloch Spherical Quantum} Genetic Algorithm. Genetic algorithms can solve the optimization problem for gradient-less information. Traditional genetic algorithms adopt binary encoding and may cause the problem of local optimum due to a single gene state. In order to effectively optimize performance, the Bloch spherical quantum genetic algorithm based on the Bloch spherical search is employed, to optimize the object function so as to determine the lower limit $x_{\mathrm{opt} i}$ of the distribution interval containing the most concentrated power points and the character power $E_{k i}$ mapping of the above interval.

\subsubsection{The Basic Principle of the Bloch Spherical Quantum} Genetic Algorithm. Genetic algorithm can solve the problem of optimization without gradient information. But traditional genetic algorithms generally adopt binary coding, which makes the gene state single and easy to get into local optimal problems.

Unlike the classical binary bit, which can only be set equal to 0 or 1 , the qubit resides in a vector space parametrized by the continuous variables $\theta$ and $\varphi$. The Bloch spherical quantum genetic algorithm utilizes Bloch spherical coordinates to encode and has an advantage on the continuous optimization problem. In order to enhance optimization efficiency and performance, we employed the method of Bloch spherical quantum genetic algorithm [24] to search the lower limit $x_{\text {opt } i}$ of the distribution interval and the corresponding character power $E_{k i}$.

Using the lower limit of the distribution interval $x$, for example, the encoded code is shown in

$$
X_{e}=\left|\begin{array}{c}
\cos \varphi_{e} \sin \theta_{e} \\
\sin \varphi_{e} \sin \theta_{e} \\
\cos \theta_{e}
\end{array}\right|,
$$

where $e=1,2, \ldots, S$ ( $S$ is the population quantity). From (15), it is known that its individuals are encoded directly by qubits described on the Bloch sphere with two parameters 


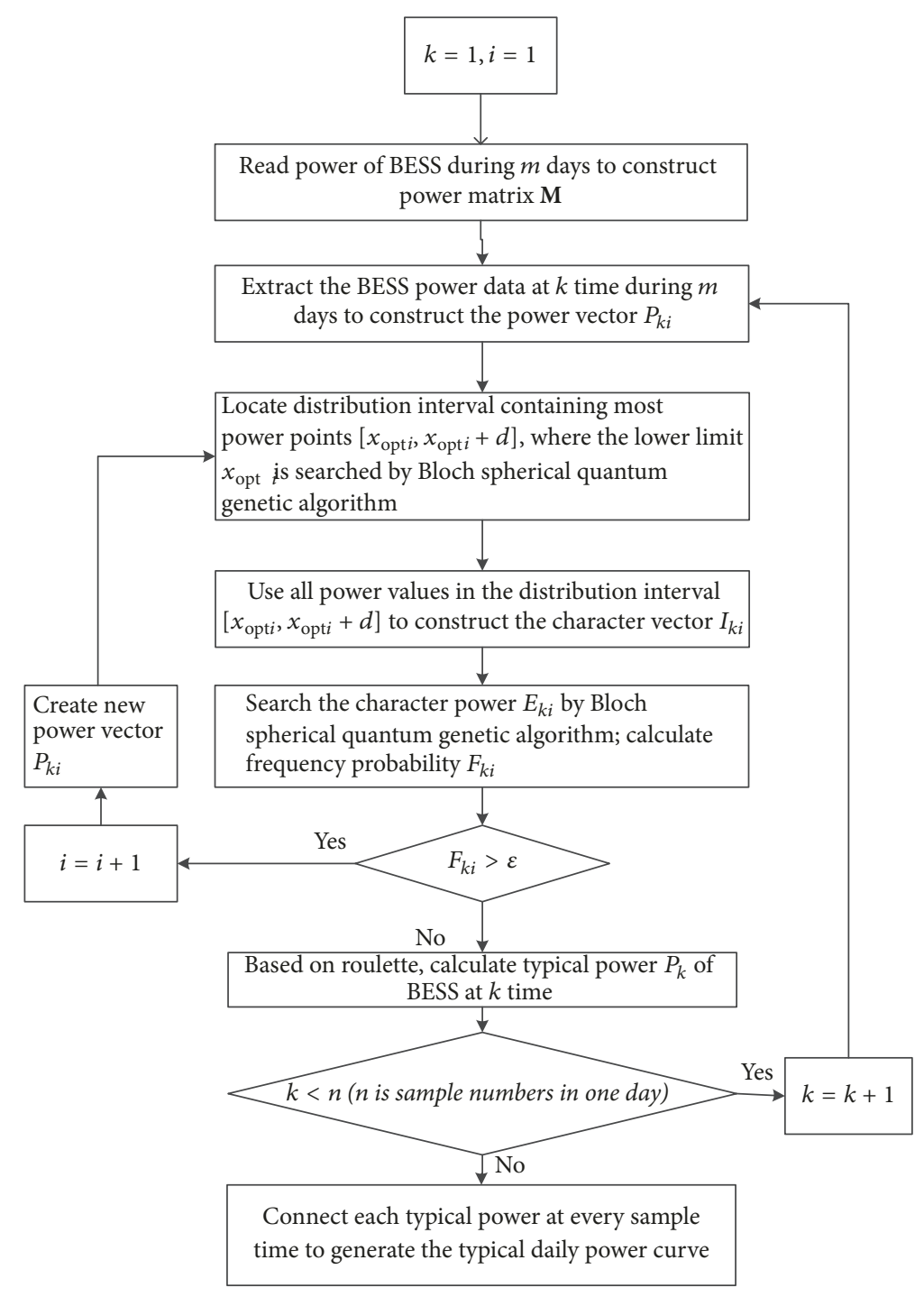

FIGURE 4: Mining algorithm for typical daily power curve of energy storage system.

$\theta$ and $\varphi$. The Bloch spherical quantum genetic algorithm uses quantum rotation gates on the Bloch sphere to update two parameters $\theta$ and $\varphi$ to accomplish the best matching of two adjustments to implement the selection and crossover of the population. In order to increase individual diversity and prevent premature convergence, the quantum nongate is used to perform the mutation.

\subsubsection{The Optimizing Implementation of the Lower Limit $x_{\text {opti }}$} of the Distribution Interval and Character Power $E_{k i}$ Mapping of the Interval. Using the lower limit $x$ of the distribution interval $L_{\text {opti }}$, for example, the optimal solution $x_{\text {opti }}$ by the Bloch spherical quantum genetic algorithm can be described in the following.

(1) Set initial parameters: the population quantity is $S$, and the maximal number of iterations is $t_{\max }$. Set the accumulation number $t=1$. Use Bloch spherical coordinates to encode the lower limit $x$, and two parameters $\theta$ and $\varphi$ are set randomly, so as to generate initial population $T(t)$.

(2) Transform solution space: the individual is processed using inverse normalization according to the range between maxima and minima of $\mathbf{P}_{\mathbf{k} \mathbf{i}}$.

(3) Utilizing (10), evaluate the fitness of each individual, and record the optimal fitness of individual and mapping parameters $\varphi$ and $\theta$; get the current optimum solution $B X$ and current optimum chromosome $B C$.

(4) Store $B X$ into the global optimum solution $G X$ and $B C$ into the global optimum solution $G C$.

(5) Set $t=t+1$, and update parameters $\varphi, \theta$ using quantum rotation gates to accomplish the selection and crossover of the population. The mutation is implemented by the quantum nongate. Therefore, a new population is generated as $T(t)$. 
(6) Iterate Steps (2) and (3), calculating the current optimum solution for $B X$ and the current optimum chromosome $B C$.

(7) If fit $(B X)<\operatorname{fit}(G X)$, then $B X \leftarrow G X, B C \leftarrow G C$. Else, $G X \leftarrow B X, G C \leftarrow B C$.

(8) Go back to (5); continue until $t>t_{\max }$. Finally, $G X$ is exported as the optimal solution $x_{\mathrm{opt} i}$.

In a similar way, the optimizing procedure for the character power $E_{k i}$ mapping of the above distribution interval is implemented by Bloch spherical quantum genetic algorithm. Equation (13) is used to evaluate the fitness of the individual during the process of searching for optimal solution for character power $E_{k_{i}}$. The individual population in inverse normalization is processed according to the range between the maxima and minima of $\mathbf{I}_{\mathbf{k i}}$.

\section{Simulation Results and Analysis}

In our simulation, actual power at a PV station with capacity power $30 \mathrm{MW}$ in China is selected to test the effectiveness of the proposed method. Sampling time is one minute.

4.1. Typical Daily Power Curve. Under the application scenario of smoothing PV power fluctuations with BESS, a smoothing strategy based on slope control [23] is utilized to obtain the operating power in BESS. Its principle can be briefed in the following:

Define the rate of power change to be calculated as

$$
r_{p}(t)=\frac{P(t)-P_{\text {smooth }}(t-\Delta t)}{\Delta t},
$$

where $P(t)$ is PV power at time $t, P_{\text {smooth }}(t-\Delta t)$ is the smoothing power at the previous sample time, and $\Delta t$ is the sample time. rules:

The smoothing power is determined using the following

(a) If $k_{\text {drop }} \leq r_{p}(t) \leq k_{\text {rise }}$, then $P_{\text {smooth }}(t)=P(t)$. $k_{\text {rise }}$.

(b) If $r_{p}(t)>k_{\text {rise }}$, then $P_{\text {smooth }}(t)=P_{\text {smooth }}(t-\Delta t)+\Delta t$.

(c) If $r_{p}(t)<k_{\text {drop }}$, then $P_{\text {smooth }}(t)=P_{\text {smooth }}(t-\Delta t)+\Delta t$. $k_{\text {drop }}$.

The above-specified parameter values $k_{\text {drop }}$ and $k_{\text {rise }}$ for the rise/drop rate limits were determined as follows:

$$
\begin{aligned}
k_{\text {rise }} & =\frac{P_{\text {rate }} \times \delta}{T} \\
k_{\text {drop }} & =-\frac{P_{\text {rate }} \times \delta}{T},
\end{aligned}
$$

where $P_{\text {rate }}$ is the capacity power of PV station and $\delta$ is the appointed limit value of power fluctuation rate during time $T$, which is generally 10 minutes.

Hence, the charging or discharging power in the BESS can be calculated as follows:

$$
P_{\text {bess }}(t)=P_{\text {smooth }}(t)-P(t)
$$

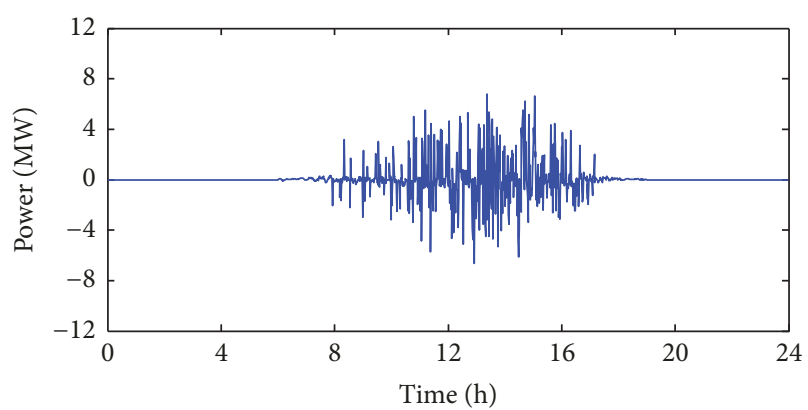

Figure 5: Typical daily power curve of the energy storage system.

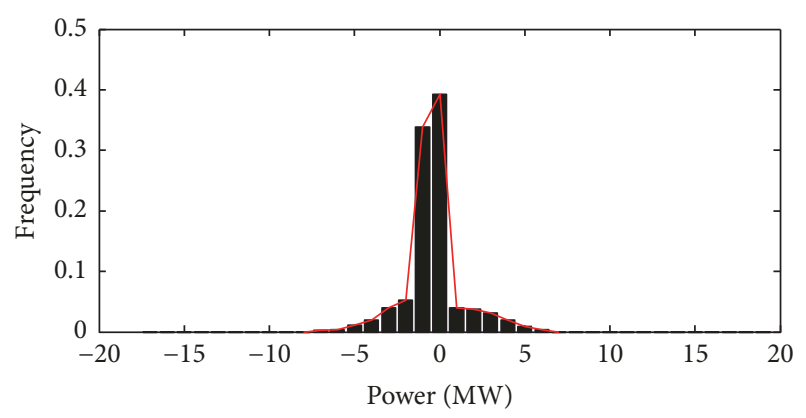

Distribution Fitting

FIGURE 6: Distribution histogram and fitting curve for the typical daily power curve.

In our simulation, $P_{\text {rate }}=30 \mathrm{MW}, T=10 \mathrm{~min}, \delta=10 \%$, and $\Delta t=1 \mathrm{~min}$. Using actual power at a PV station, the charging/discharging power in BESS can be obtained by (15). Furthermore, implementing the proposed mining algorithm in the paper, the typical daily power curve of BESS can be mined as shown in Figure 5. The distribution histogram and fitting curve of the BESS typical daily power curve are given in Figure 6.

The typical power curve of energy storage system explores operation data and is the refinement and generalization of actual power, which can reflect the charge and discharge characteristics of energy storage system. Mining knowledge from the typical power curve will help to better utilize BESS. Based on typical power curve, decision-maker can master the operating conditions of system and increase the overall cognition of output power of energy storage. According to the curve data in Figure 5, the operation parameters can be extracted as Table 1 . The maximum charging power, maximum discharging power, maximum power fluctuation rate, and charging capacity and discharging capacity in BESS during one day could be found in Table 1. It is valuable to optimize control of BESS performance to reduce the economic cost of BESS through analyzing those important operation parameters.

By using the typical power curve, it can also help determine the optimal capacity allocation of BESS. In order to get suitable capacity allocation of BESS, long time span data for one year is commonly needed to calculate the capacity 
TABLE 1: Operation parameters in the typical power curve.

\begin{tabular}{lcccc}
\hline $\begin{array}{l}\text { Maximum charging power } \\
(\mathrm{MW})\end{array}$ & $\begin{array}{c}\text { Maximum discharging } \\
\text { power } \\
(\mathrm{MW})\end{array}$ & $\begin{array}{c}\text { Maximum power } \\
\text { fluctuation rate } \\
(\mathrm{MW})\end{array}$ & $\begin{array}{c}\text { Charging capacity } \\
(\mathrm{MWh})\end{array}$ & $\begin{array}{c}\text { Discharging capacity } \\
(\mathrm{MWh})\end{array}$ \\
\hline 6.81 & 6.65 & 10.15 & 0.36 & 0.28 \\
\hline
\end{tabular}

TABLE 2: Vertical error $I_{R}$.

\begin{tabular}{lcccccc}
\hline Weather pattern & Sunny & Cloudy & Overcast & Rain/snow & Cloudy to sunny & Sunny to cloudy \\
\hline $\mathbf{I}_{\mathbf{R}}$ & 1.18 & 0.85 & 1.02 & 1.20 & 1.13 & 0.83 \\
\hline
\end{tabular}

allocation of BESS. Using the extracted typical power curve to calculate optimal capacity allocation of BESS, it can effectively compress the data and cut down the amount of calculation. lows:

The calculation of optimal capacity allocation is as fol-

$$
\begin{aligned}
& \text { Cap }=\max \left(N_{1}, N_{2}\right) \\
& N_{1}=\max \left(\left|P_{1} \Delta t\right|,\left|P_{1} \Delta t+P_{2} \Delta t\right|, \ldots,\right. \\
& \left.\quad\left|P_{1} \Delta t+P_{2} \Delta t+\cdots+P_{n} \Delta t\right|\right) \\
& N_{2}=\max \left(\left|\sum_{i=1}^{m_{1}} P_{i} \Delta t\right|,\left|\sum_{i=m_{2}}^{m_{3}} P_{i} \Delta t\right|, \ldots,\left|\sum_{i=m_{j}}^{m_{n}} P_{i} \Delta t\right|\right),
\end{aligned}
$$

where Cap is the capacity allocation of BESS, $P_{i}$ is the power value of the typical power curve at time $I, \Delta t$ is the sample interval, and $1 \sim m_{1}, m_{2} \sim m_{3}, m_{j} \sim m_{n}$ are the sample times that the BESS continuously charge and discharge.

Substituting the power value of the typical power curve into the above calculation formula can be used to obtain the optimal capacity allocation of BESS. The optimal capacity allocation achieved, based on typical power curve, is $2.71 \mathrm{MW} \cdot \mathrm{h}$. Thus, the result of the capacity allocation of BESS for the above PV station is $6.81 \mathrm{MW} / 2.71 \mathrm{MW} \cdot \mathrm{h}$, according to the mined typical power curve.

4.2. Error Evaluation. In order to verify the effectiveness of the mined typical power curve, an error evaluation is presented between the typical power curve and power curves in different weather patterns. A vertical error index and probability distribution error are designed.

\subsubsection{Vertical Error $I_{R}$. We define the vertical error $I_{R}$ as}

$$
\begin{aligned}
& I_{R}(l)=\sqrt{n^{-1} \sum_{i=1}^{n}\left[\frac{P_{\text {typical }}(i)-P_{l}(i)}{Q_{l}(i)}\right]^{2}} \\
& Q_{l}(i)= \begin{cases}1, & \left|P_{l}(i)\right|<1 \\
P_{l}(i), & \text { else, }\end{cases}
\end{aligned}
$$

where $I_{R}(l)$ represents the vertical error between the typical power curve and power curve in the weather pattern $l$, $P_{\text {typcial }}(i)$ is the $i$ th power in the typical power curve, $P_{l}(i)$ is the $i$ th power in the weather pattern $l$, and $n$ is the number of power points.

The vertical error $I_{R}(l)$ measures the dispersion degree between the typical power curve and the power curves in different weather. It is an error evaluation index for the typical power curve from the time domain.

The mined typical power curve and power curves under the six weather patterns are shown in Figure 8. Table 2 shows the vertical errors between the typical power curve and the power curve in BESS for the case of six weather patterns.

It can be seen that, from Table 2 and Figure 7, the typical power curve implies the characteristics of power under different weather. The vertical errors between the typical power curve and the power curve are relatively larger under two weather conditions (sunny and rain/snow) than the other four weather patterns. This is due to the fact that, on sunny and rain/snow days, the power fluctuation of the PV station is small, resulting in a low requirement for stored power in BESS. Thus the amplitude of the BESS power curve is small and smooth, compared with the other four weather patterns. This results in heavy dispersion from the typical power curve.

4.2.2. Probability Distribution Error $I_{F}$. We define the evaluating index $I_{F}$ as

$$
I_{F}(l)=d^{-2} \sum_{i=1}^{n}\left|f_{\text {typical }}(i)-f_{l}(i)\right|
$$

where $I_{F}(l)$ represents the error of fitting curve of the probability density between the typical power curve and BESS power curve in the weather pattern $l$. We define $f_{\text {typcial }}(i)$ as the $i$ th value in the fitting curve for typical power curve. Also, $f_{l}(i)$ is the $i$ th value in the fitting curve for the weather pattern $l, n$ is the number of power points, and $d$ is the width of bin in the histogram.

The fitting curves of the probability density for both the typical daily power curve and power curves under the six weather patterns are shown in Figure 8 . Table 3 presents the probability distribution error of the fitting curves between the typical power curve and others.

Table 3 shows that there are small probability distribution errors for the fitting curve between the typical daily power curve and power curves under the six weather patterns. The fitting curve of the typical daily power curve represents a similar trend to others. In comparison with the other five weather patterns, the probability distribution error is 
TABle 3: Probability distribution error $I_{F}$.

\begin{tabular}{lcccccc}
\hline Weather pattern & Sunny & Cloudy & Overcast & Rain/snow & Cloudy to sunny & Sunny to cloudy \\
\hline$I_{F}$ & 0.69 & 0.57 & 0.52 & 0.45 & 0.48 & 0.38 \\
\hline
\end{tabular}

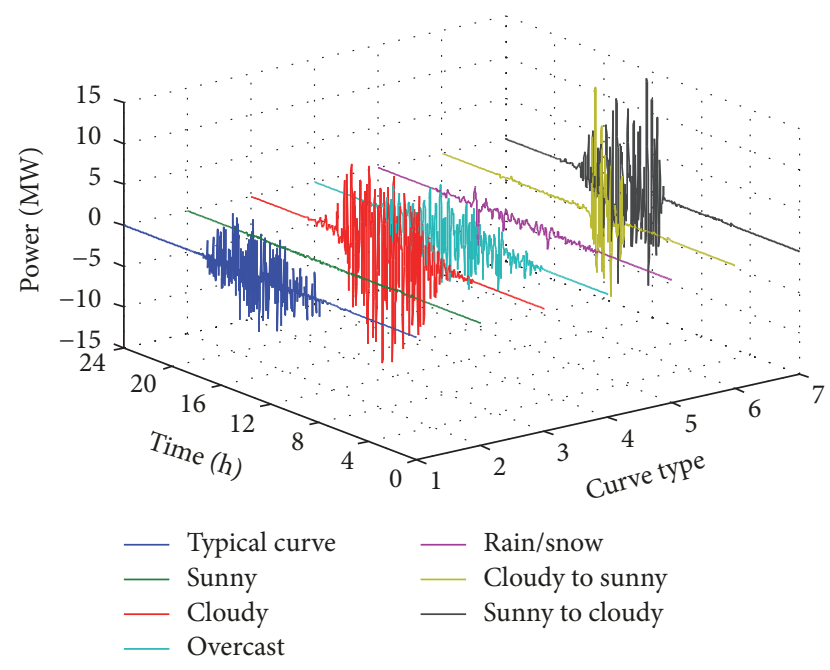

Figure 7: Typical power curve and power curves under six typical weather patterns.

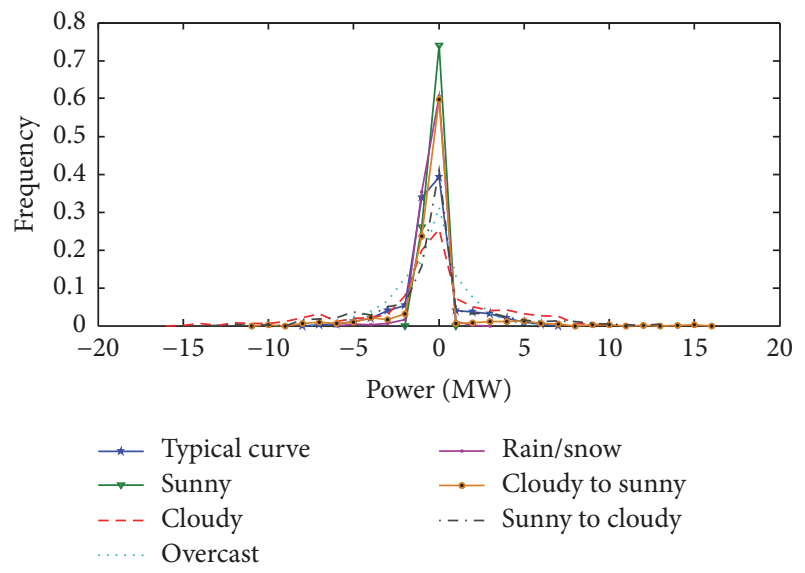

FIGURE 8: Distribution fitting curves for both typical power and others.

relatively larger between the typical daily power curve and the power curve on the sunny day. In our view, the typical power curve is extracted from synthesizing the power information under the case of six weather patterns. The fitting curve of the probability density on a sunny day mainly centralizes near zero and is different from other curves. It results in relatively large probability distribution errors between the typical power curve and sunny power curve.

\section{Conclusions}

In this work, a new method of mining the typical daily power curve for BESS is proposed under the application scenario of smoothing PV power fluctuations. Since the BESS is a potential technology for solving the problem of high penetration in PV power stations and BESS is expensive, it is valuable that a typical daily power curve is extracted, to discover the operating feature in BESS. The operational characteristics of charging/discharging power in BESS under different weather patterns are analyzed by applying fuzzy$c$ means clustering techniques. Due to dispersed power data, a novel mining algorithm is proposed that utilizes the probability distribution information of data in the vertical time series and Bloch spherical quantum genetic search algorithm to extract the typical power value for sample times. For every sample time, based on the probability distribution of power in the vertical time series, a group of the distribution intervals containing more power points are searched by the Bloch spherical quantum genetic algorithm. The character power mapping of every distribution interval is also determined from the scattered data using the Bloch spherical quantum genetic algorithm. In order to maintain the diversity of power, the typical power value at every sample time is further generated by applying the principle of roulette according to the probability of character power. Finally, a typical daily power curve for BESS is achieved by connecting the overall typical power value at each sample time. According to the error-evaluating index, the mined typical power curve contains features of the operating power of BESS under different weather patterns and shows the useful charging/discharging power information effectively. It will be helpful for the decision-maker to increase the overall cognition of operating conditions of energy storage and further optimize control performance and capacity allocation to reduce the economic cost of BESS.

\section{Nomenclature}

PV: Photovoltaic

BESS: Battery energy storage system

FCM: Fuzzy- $C$ means

$\mathrm{X}$ : Input data to be clustered

V: The matrix of cluster centers

$C$ : The total number of clusters

$\mu_{c}(i)$ : Membership degree of the $i$ th input data to center of the $C$ th cluster

$V_{1 i}$ : The irradiance fluctuation rate before 12 hours of the $i$ th day

$V_{2 i}$ : The irradiance fluctuation rate after 12 hours of the $i$ th day

$T_{i}$ : $\quad$ Average temperature of the $i$ th day 
$K_{i}: \quad$ The maximum irradiance ratio of the $i$ th day

$I_{i}$ : $\quad$ Actual solar irradiance received of PV panel of the $i$ th day

$E_{i}^{c}$ : $\quad$ Maximum irradiance in external horizontal plane of the $i$ th day

$V_{c}$ : $\quad$ Cluster center of the $C$ th cluster

$k$ : $\quad$ Sample time

$P_{k}$ : The typical power value at time $k$

M: $\quad$ Power matrix

$m$ : Number of days of historical data

$n$ : $\quad$ Sample points for one day

$\mathbf{P}_{k 1}$ : Power vector that is made up of $m$ powers at sample time $k$

$L_{\text {opt1 }}$ : The distribution interval containing the most concentrated power points

$x$ : $\quad$ The lower limit of the interval

$d$ : The range of interval

$x_{\text {opt } 1}$ : The lower limit of the distribution interval containing the most concentrated power points

$\mathbf{I}_{k 1}$ : The interval power vector

$p_{i k}^{\prime}$ : The power value in $L_{\text {optl }}$

$F_{k 1}$ : The frequency probability of distribution interval

$t$ : The size of the interval power vector

$E_{k 1}$ : Character power mapping in first interval

$\mathbf{P}_{k 2}$ : Power vector obtained by removing $\mathbf{I}_{k 1}$ from $\mathbf{P}_{k 1}$

$L_{\text {opt2 }}$ : Next optimal distribution interval

$\mathbf{I}_{k 2}$ : The second interval power vector

$F_{k 2}$ : The frequency probability of second interval

$\varepsilon$ : The threshold of frequency probability

$x_{\text {opt } i}$ : The lower limit of the $i$ th distribution interval containing the most concentrated power points

$F_{k i}: \quad$ The frequency probability of $i$ th interval

$E_{k i}$ : Character power mapping in $i$ th interval

$\mathbf{E}_{k}$ : Typical character power vector

$X_{e}$ : The encoded code of parameter $x$ by Bloch spherical quantum genetic algorithm

S: $\quad$ The population quantity

$\theta$ : $\quad$ Parameter variable of Bloch sphere

$\varphi$ : $\quad$ Parameter variable of Bloch sphere

$t_{\text {max }}$ : The maximal number of iterations

$T(t)$ : Initial population

$B X$ : Current optimum solution

$B C$ : Current optimum chromosome.

\section{Conflicts of Interest}

The authors declare no conflicts of interest.

\section{Acknowledgments}

The authors would like to acknowledge the funding support from National Nature Science Fund Project (51677067),
Science and Technology Project of SGCC (DG71-14-046), and Beijing New-Star Plan of Science and Technology (Z141101001814094).

\section{References}

[1] G. S. Kinsey, A. Nayak, M. Liu, V. Garboushian, and S. Beach, "Increasing power and energy in amonix solar power plants," IEEE J. Photovoltaics, vol. 1, pp. 3-4, 2011.

[2] M. Huber, D. Dimkova, and T. Hamacher, "Integration of wind and solar power in Europe: Assessment of flexibility requirements," Energy, vol. 69, pp. 236-246, 2014.

[3] S. Hashemi, J. Ostergaard, and G. Yang, "A scenario-based approach for energy storage capacity determination in LV grids with high PV penetration," IEEE Transactions on Smart Grid, vol. 5, no. 3, pp. 1514-1522, 2014.

[4] F. Geth, T. Brijs, J. Kathan, J. Driesen, and R. Belmans, "An overview of large-scale stationary electricity storage plants in Europe: Current status and new developments," Renewable \& Sustainable Energy Reviews, vol. 52, pp. 1212-1227, 2015.

[5] N. Jayasekara, P. Wolfs, and M. A. S. Masoum, "An optimal management strategy for distributed storages in distribution networks with high penetrations of PV," Electric Power Systems Research, vol. 116, pp. 147-157, 2014.

[6] S. Wogrin and D. F. Gayme, "Optimizing Storage Siting, Sizing, and Technology Portfolios in Transmission-Constrained Networks," IEEE Transactions on Power Systems, vol. 30, no. 6, pp. 3304-3313, 2015.

[7] M. Bortolini, M. Gamberi, and A. Graziani, “Technical and economic design of photovoltaic and battery energy storage system," Energy Conversion and Management, vol. 86, pp. 81-92, 2014.

[8] B. Battke, T. S. Schmidt, D. Grosspietsch, and V. H. Hoffmann, "A review and probabilistic model of lifecycle costs of stationary batteries in multiple applications," Renewable \& Sustainable Energy Reviews, vol. 25, pp. 240-250, 2013.

[9] Y. Riffonneau, S. Bacha, F. Barruel, and S. Ploix, "Optimal power flow management for grid connected PV systems with batteries," IEEE Transactions on Sustainable Energy, vol. 2, no. 3, pp. 309-320, 2011.

[10] X. Liu, A. Aichhorn, L. Liu, and H. Li, "Coordinated control of distributed energy storage system with tap changer transformers for voltage rise mitigation under high photovoltaic penetration," IEEE Transactions on Smart Grid, vol. 3, no. 2, pp. 897-906, 2012.

[11] M. Z. Daud, A. Mohamed, and M. A. Hannan, "An improved control method of battery energy storage system for hourly dispatch of photovoltaic power sources," Energy Conversion and Management, vol. 73, pp. 256-270, 2013.

[12] A. Nottrott, J. Kleissl, and B. Washom, "Energy dispatch schedule optimization and cost benefit analysis for grid-connected, photovoltaic-battery storage systems," Journal of Renewable Energy, vol. 55, pp. 230-240, 2013.

[13] D. Gerbec, S. Gašperič, I. Šmon, and F. Gubina, "Determining the load profiles of consumers based on fuzzy logic and probability neural networks," IEE Proceedings Generation, Transmission and Distribution, vol. 151, no. 3, pp. 395-400, 2004.

[14] H. S. Hippert, E. C. Pedreira, and C. R. Souza, "Neural networks for short-term load forecasting: a review and evaluation," IEEE Transactions on Power Systems, vol. 16, no. 1, pp. 44-55, 2001. 
[15] S. Rahman and R. Bhatnagar, "An expert system based algorithm for short term load forecast," IEEE Transactions on Power Systems, vol. 3, no. 2, pp. 392-399, 1988.

[16] K.-L. Zhou, S.-L. Yang, and C. Shen, "A review of electric load classification in smart grid environment," Renewable \& Sustainable Energy Reviews, vol. 24, pp. 103-110, 2013.

[17] G. Chicco, "Overview and performance assessment of the clustering methods for electrical load pattern grouping," Energy, vol. 42, no. 1, pp. 68-80, 2012.

[18] G. J. Tsekouras, N. D. Hatziargyriou, and E. N. Dialynas, “Twostage pattern recognition of load curves for classification of electricity customers," IEEE Transactions on Power Systems, vol. 22, no. 3, pp. 1120-1128, 2007.

[19] J. W. Taylor and P. E. McSharry, "Short-term load forecasting methods: An evaluation based on European data," IEEE Transactions on Power Systems, vol. 22, no. 4, pp. 2213-2219, 2007.

[20] S.-J. Huang and K.-R. Shih, "Short-term load forecasting via ARMA model identification including non-Gaussian process considerations," IEEE Transactions on Power Systems, vol. 18, no. 2, pp. 673-679, 2003.

[21] V. Dordonnat, S. J. Koopman, M. Ooms, A. Dessertaine, and J. Collet, "An hourly periodic state space model for modelling French national electricity load," International Journal of Forecasting, vol. 24, no. 4, pp. 566-587, 2008.

[22] M. Mosadeghy, R. Yan, and T. K. Saha, "Impact of PV penetration level on the capacity value of South Australian wind farms," Journal of Renewable Energy, vol. 85, pp. 1135-1142, 2016.

[23] X. Li, D. Hui, and X. Lai, "Battery energy storage station (BESS)-based smoothing control of photovoltaic (PV) and wind power generation fluctuations," IEEE Transactions on Sustainable Energy, vol. 4, no. 2, pp. 464-473, 2013.

[24] P. Li and S. Li, "Quantum-inspired evolutionary algorithm for continuous space optimization based on Bloch coordinates of qubits," Neurocomputing, vol. 72, no. 1-3, pp. 581-585, 2008. 


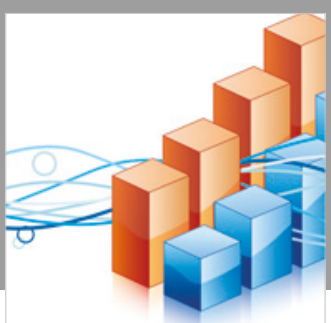

Advances in

Operations Research

\section{-n-m}
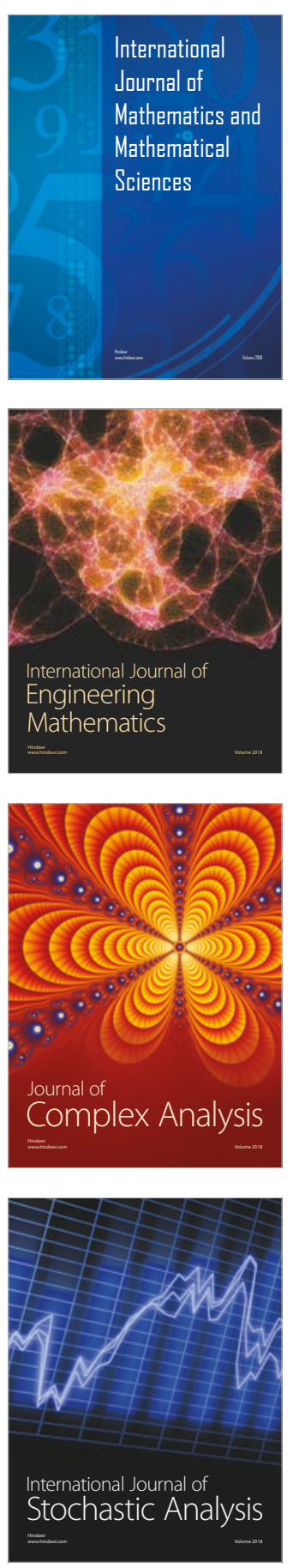
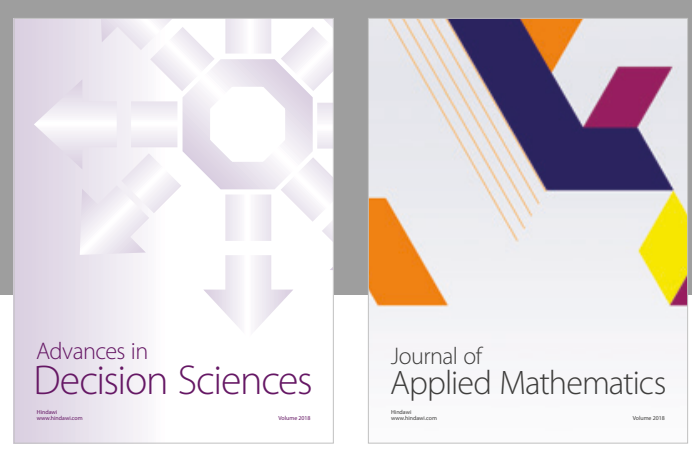

Journal of

Applied Mathematics
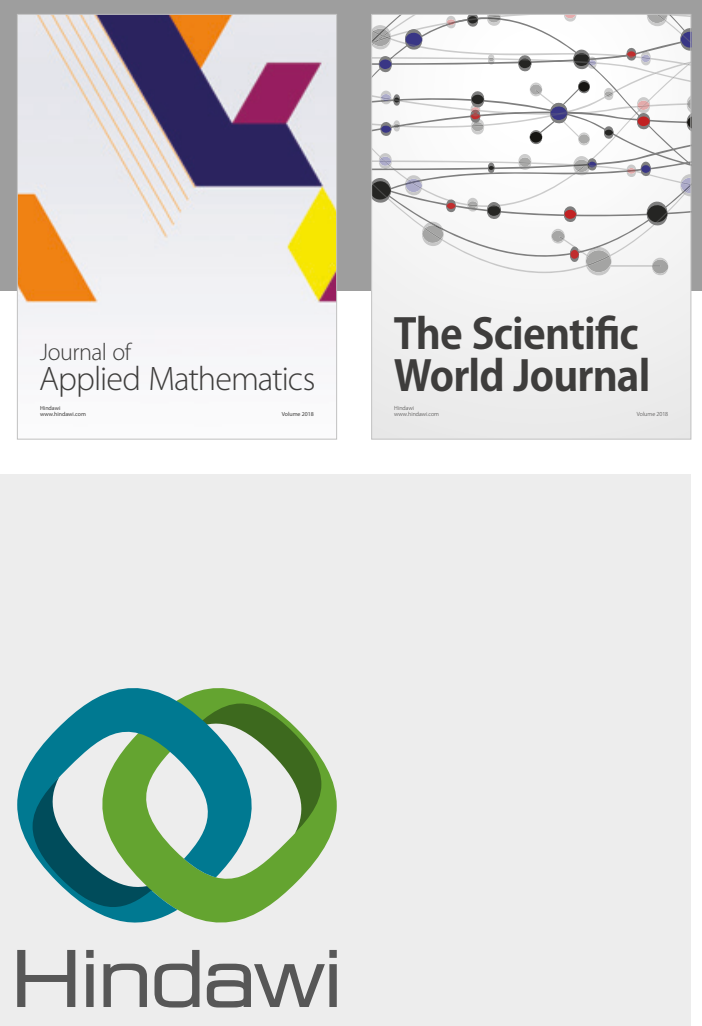

Submit your manuscripts at

www.hindawi.com

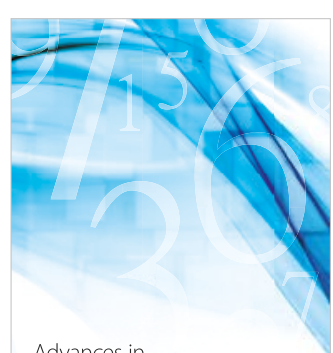

Advances in
Numerical Analysis
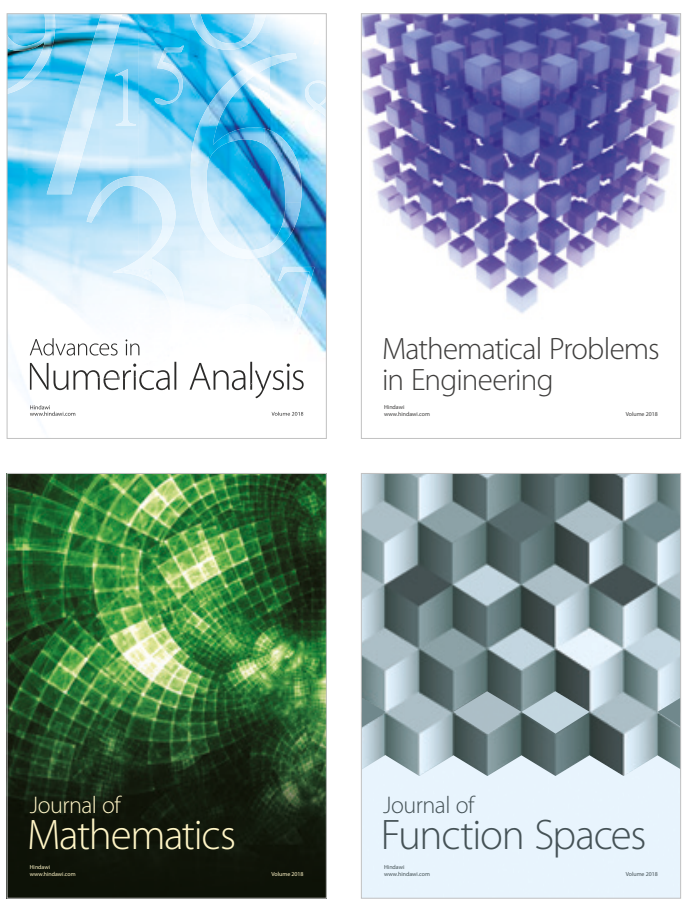

Mathematical Problems in Engineering

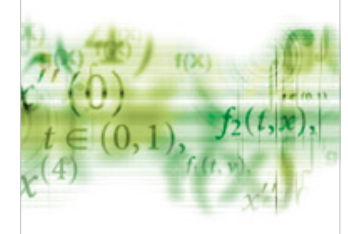

International Journal of

Differential Equations

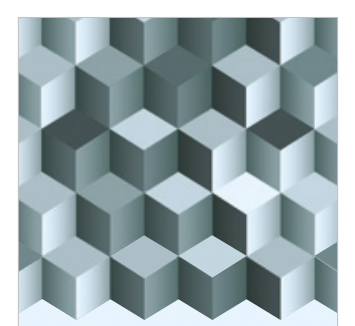

Journal of

Function Spaces

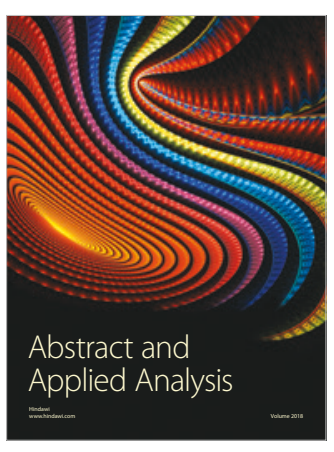

The Scientific

World Journal

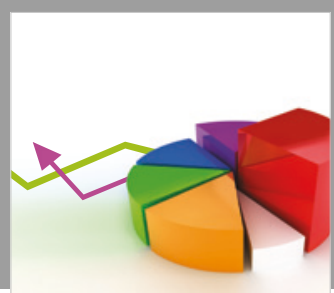

Journal of

Probability and Statistics
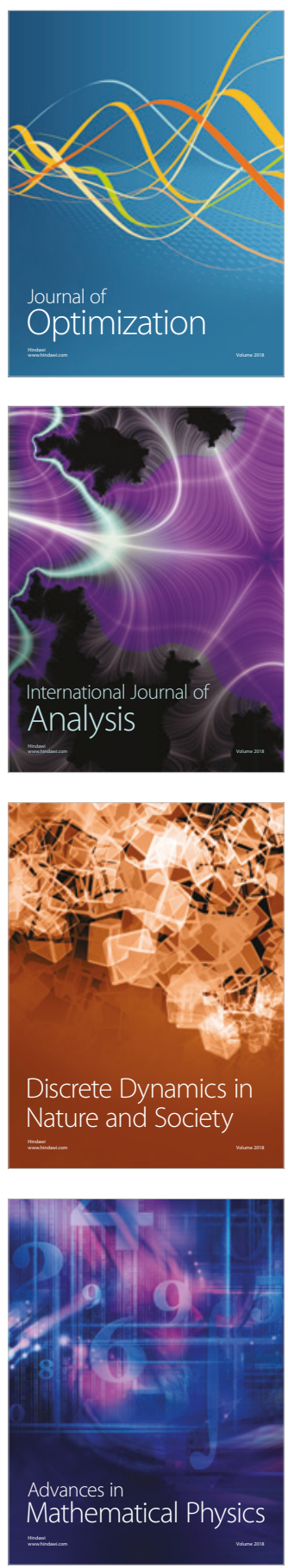